\title{
Non-exponential length dependence of conductance in iodide- terminated oligothiophene single-molecule tunneling junctions
}

Limin Xiang $\dagger,{ }^{1,3}$ Thomas Hines $\dagger,{ }^{1}$ Julio L. Palma, ${ }^{1}$ Xuefeng Lu, ${ }^{2}$ Vladimiro Mujica, ${ }^{3}$ Mark A. Ratner, ${ }^{4}$ Gang $\mathrm{Zhou}^{2 *}$, and Nongjian Tao ${ }^{1,5 *}$

${ }^{1}$ Center for Biosensors and Bioelectronics, Biodesign Institute, Arizona State University, Tempe, Arizona 85287, USA.

${ }^{2}$ Laboratory of Advanced Materials, Fudan University, Shanghai 200438, P.R. China.

${ }^{3}$ School of Molecular Sciences, Arizona State University, Tempe, Arizona 85287, USA.

${ }^{4}$ Department of Chemistry, Northwestern University, Evanston, Illinois 60208, USA.

${ }^{5}$ School of Electrical, Computer and Energy Engineering, Arizona State University, Tempe, Arizona 85287, USA.

Email: zhougang@fudan.edu.cn; Nongjian.Tao@asu.edu

*Corresponding Authors

$\dagger$ Contributed equally to this work 
Table of Contents:

1. Synthesis and ${ }^{1} \mathrm{H}$ NMR spectrum of Compounds IT-n $(n=1,2,3,4,5,6)$..............S3-8

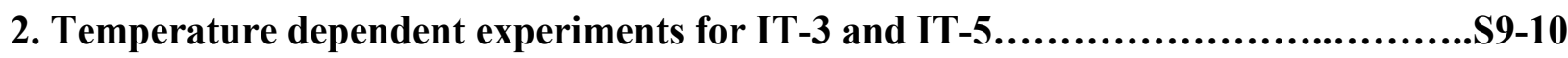

3. Data collections, 2-Dimensional GV (conductance-voltage, $G=I / V$ ) and TVS (Transition voltage spectroscopy) Histograms..............................................S10-14

4. Control experiment in mesitylene.....................................................S15

5. XPS analysis of IT-1 and mesitylene on a Au surface...............................

6. Discussions on the possibility of C-I bond cleavage for IT-n......................S16-18

7. Conductance measurement under nitrogen atmosphere for IT-2.....................S19

8. Energy barrier for Rotation of Thiophene Rings.................................S19-20

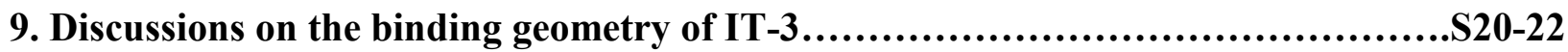


1. Synthesis and ${ }^{1} \mathrm{H}$ NMR spectrum of Compounds IT-n $(\mathrm{n}=1,2,3,4,5,6)$.

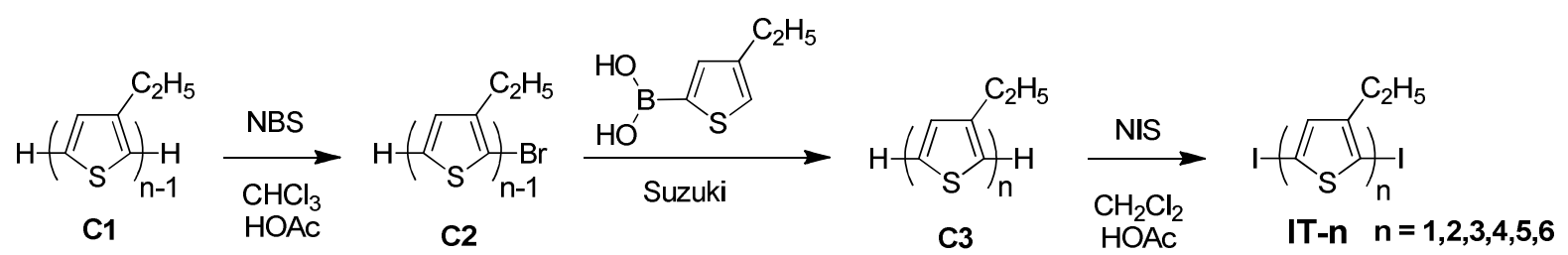

Figure S1a. Synthetic Route for Compounds IT-n

General Synthesis Procedure for Compound C2. Under a nitrogen atmosphere, C1 (10 mmol) was dissolved in a solution of chloroform, $N$-bromosuccinimide (NBS, $1.78 \mathrm{~g}, 10 \mathrm{mmol}$ ) was added slowly at $0{ }^{\circ} \mathrm{C}$, After the mixture was stirred at room temperature for $6 \mathrm{~h}$, distilled water was added to quench the reaction. The solution was extracted with dichloromethane (DCM), washed with sodium hydroxide solution and brine and dried over anhydrous $\mathrm{Na}_{2} \mathrm{SO}_{4}$. After removal of the solvent, the bromide $\mathbf{C} \mathbf{2}$ was obtained by flash column chromatography.

General Synthesis Procedure for Compound C3. In a flask, a mixture of C2 (10 mmol), 4ethylthienylboronic acid (1.87g, $12 \mathrm{mmol}), \mathrm{Pd}\left(\mathrm{PPh}_{3}\right)_{4}(80 \mathrm{mg}, 0.07 \mathrm{mmol})$ and $\mathrm{K}_{2} \mathrm{CO}_{3}(2.76 \mathrm{~g}$, $0.02 \mathrm{~mol})$ in a solution of water $(10 \mathrm{~mL})$ and THF $(20 \mathrm{~mL})$ was stirred and heated at $80{ }^{\circ} \mathrm{C}$ for 12 h. When the reaction was completed, the mixture was extracted with DCM and washed with brine and dried by anhydrous $\mathrm{Na}_{2} \mathrm{SO}_{4}$. Concentrated by rotary evaporation, the residue was purified by flash column chromatography and yielded $\mathbf{C 3}$.

General Synthesis Procedure for Compounds IT-n. C3 (1 mmol) was dissolved in a solution of DCM and cooled to $0{ }^{\circ} \mathrm{C}$, then $N$-iodosuccinimide (NIS, $495 \mathrm{mg}, 2.2 \mathrm{mmol}$ ) was added in portions. Glacial acetic acid $(0.38 \mathrm{~mL}, 6.6 \mathrm{mmol})$ was added dropwise via syringe. The ice bath was removed, and the reaction mixture was allowed to stir at room temperature overnight. The 
mixture was extracted with DCM, washed with sodium hydroxide solution and brine and dried over anhydrous $\mathrm{Na}_{2} \mathrm{SO}_{4}$. Concentrated by rotary evaporation, and separated on a silica column. The desired compounds IT-n were obtained.

Compound IT-1. ${ }^{1} \mathrm{H}$ NMR (400 MHz, $\mathrm{CDCl}_{3}, \delta$ ppm): $6.92(\mathrm{~s}, 1 \mathrm{H}), 2.57-2.51(\mathrm{~m}, 2 \mathrm{H}), 1.16(\mathrm{t}$, $3 \mathrm{H}, J=7.6 \mathrm{~Hz}) .{ }^{13} \mathrm{C} \mathrm{NMR}\left(100 \mathrm{MHz}, \mathrm{CDCl}_{3}, \delta \mathrm{ppm}\right): 150.88,137.64,76.83,76.24,25.74$, 14.73. MALDI-TOF Mass: $\mathrm{m} / z$ 363, calc. 363.8 .

Compound IT-2. ${ }^{1} \mathrm{H}$ NMR $\left(400 \mathrm{MHz}, \mathrm{CDCl}_{3}, \delta \mathrm{ppm}\right): 7.08(\mathrm{~s}, 1 \mathrm{H}), 6.73(\mathrm{~s}, 1 \mathrm{H}), 2.73-2.68(\mathrm{~m}$, 2H), 2.59-2.53 (m, 2H), 1.24-1.19 (m, 6H). $\left.{ }^{13} \mathrm{C} \mathrm{NMR} \mathrm{(100} \mathrm{MHz,} \mathrm{CDCl}_{3}, \delta \mathrm{ppm}\right):$ 149.04, $143.15,139.71,139.45,136.06,126.69,74.34,72.37,26.07,22.39,15.35,14.71$. MALDI-TOF Mass: $m / z$ 474, calc. 473.8 .

Compound IT-3. ${ }^{1} \mathrm{H}$ NMR (400 MHz, $\mathrm{CDCl}_{3}, \delta$ ppm): 7.09 (s, $\left.1 \mathrm{H}\right), 6.89(\mathrm{~s}, 1 \mathrm{H}), 6.80(\mathrm{~s}, 1 \mathrm{H})$, 2.79-2.71 (m, 4H), 2.60-2.54 (m, 2H), 1.29-1.20 (m, 9H). $\left.{ }^{13} \mathrm{C} \mathrm{NMR} \mathrm{(100} \mathrm{MHz,} \mathrm{CDCl}_{3}, \delta \mathrm{ppm}\right)$ : $149.07,142.85,141.58,140.51,139.60,136.32,133.28,130.49,128.62,126.26,73.86,71.94$, 26.06, 22.76, 22.49, 15.26, 15.24, 14.69. MALDI-TOF Mass: $m / z$ 584, calc. 583.9.

Compound IT-4. ${ }^{1} \mathrm{H}$ NMR (400 MHz, $\mathrm{CDCl}_{3}, \delta$ ppm): 7.10 (s, 1H), 6.97 (s, 1H), $6.91(\mathrm{~s}, 1 \mathrm{H})$, $6.82(\mathrm{~s}, 1 \mathrm{H}), 2.83-2.73(\mathrm{~m}, 6 \mathrm{H}), 2.61-2.55(\mathrm{~m}, 2 \mathrm{H}), 1.31-1.21(\mathrm{~m}, 12 \mathrm{H}) .{ }^{13} \mathrm{C}$ NMR $(100 \mathrm{MHz}$, $\left.\mathrm{CDCl}_{3}, \delta \mathrm{ppm}\right): 149.07,142.79,141.64,141.33,140.70,139.61,136.46,134.10,133.03,130.75$, $130.19,128.78,128.23,126.13,73.72,71.85,26.07,22.90,22.81,22.52,15.27,15.19,14.70$. MALDI-TOF Mass: $m / z$ 694, calc. 693.9.

Compound IT-5. ${ }^{1} \mathrm{H}$ NMR (400 MHz, $\mathrm{CDCl}_{3}, \delta$ ppm): 7.09 (s, 1H), 6.98 (s, 2H), $6.91(\mathrm{~s}, 1 \mathrm{H})$, $6.82(\mathrm{~s}, 1 \mathrm{H}), 2.84-2.73(\mathrm{~m}, 8 \mathrm{H}), 2.60-2.55(\mathrm{~m}, 2 \mathrm{H}), 1.30-1.20(\mathrm{~m}, 15 \mathrm{H}) .{ }^{13} \mathrm{C} \mathrm{NMR}(100 \mathrm{MHz}$, 
$\left.\mathrm{CDCl}_{3}, \delta \mathrm{ppm}\right): 149.06,142.77,141.65,141.41,141.27,140.73,139.60,136.48,134.28,133.84$ $132.93,130.88,130.43,130.05,128.79,128.39,128.11,126.10,73.65,71.79,26.06,22.93$, 22.91, 22.80, 22.50, 15.26, 15.20, 15.17, 14.68. MALDI-TOF Mass: $\mathrm{m} / z$ 804, calc. 803.9.

Compound IT-6. ${ }^{1} \mathrm{H}$ NMR (400 MHz, $\mathrm{CDCl}_{3}, \delta$ ppm): 7.09 (s, 1H), 6.99 (s, 3H), 6.91 (s, 1H), $6.82(\mathrm{~s}, 1 \mathrm{H}), 2.83-2.75(\mathrm{~m}, 10 \mathrm{H}), 2.60-2.54(\mathrm{~m}, 2 \mathrm{H}), 1.31-1.22(\mathrm{~m}, 18 \mathrm{H}) .{ }^{13} \mathrm{C}$ NMR $(100 \mathrm{MHz}$, $\left.\mathrm{CDCl}_{3}, \delta \mathrm{ppm}\right): 149.06,142.77,141.65,141.42,141.34,141.25,140.75,139.60,134.34,134.04$, 133.76, 132.91, 130.92, 130.57, 130.31, 130.01, 128.80, 128.41, 128.28, 128.08, 126.10, 73.63, 71.76, 29.93, 26.06, 22.94, 22.80, 22.50, 15.24, 15.18, 14.66. MALDI-TOF Mass: $m / z$ 914, calc. 913.9. 


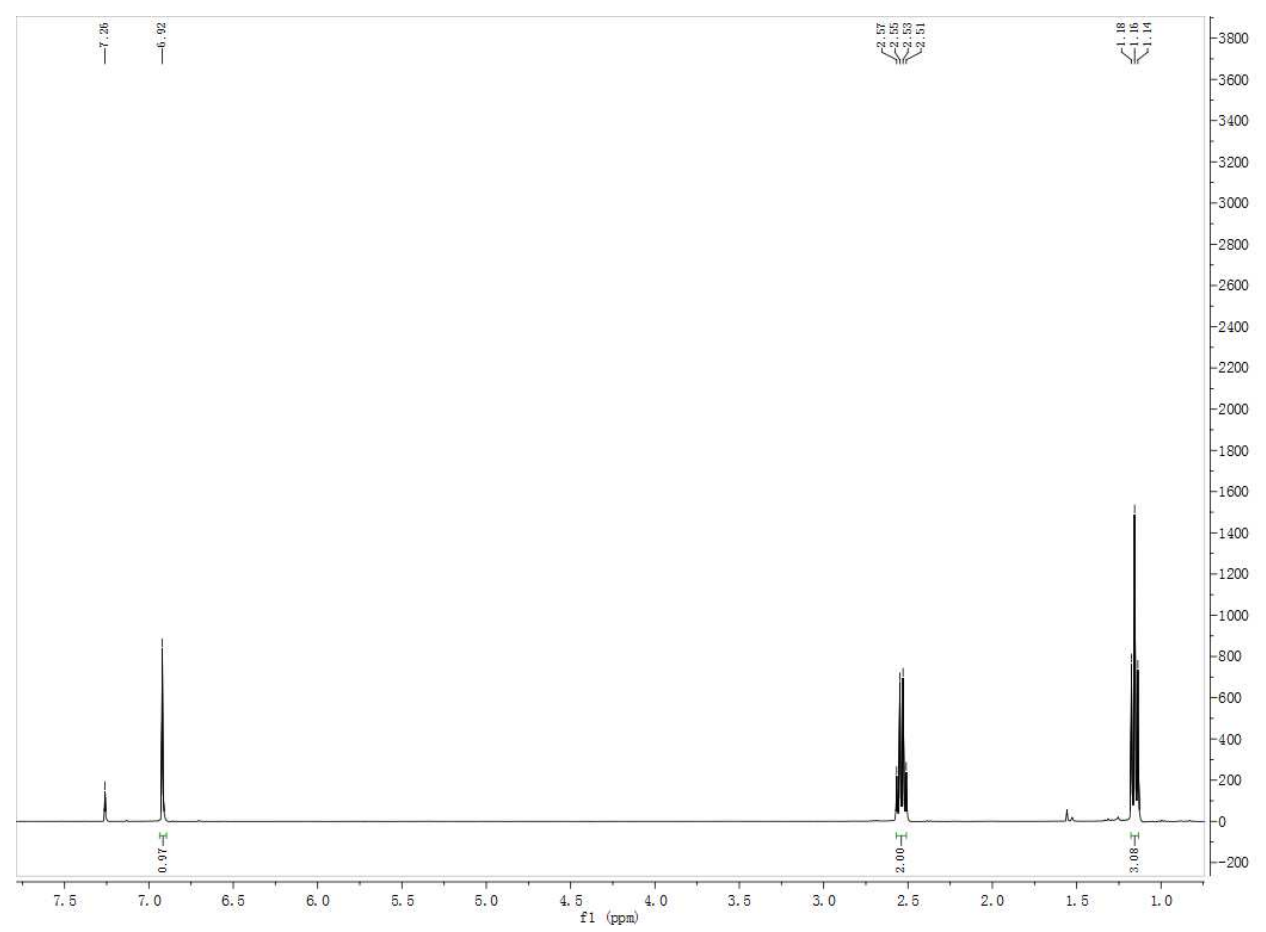

Figure S1b. ${ }^{1} \mathrm{H}$ NMR spectrum of compound IT-1.

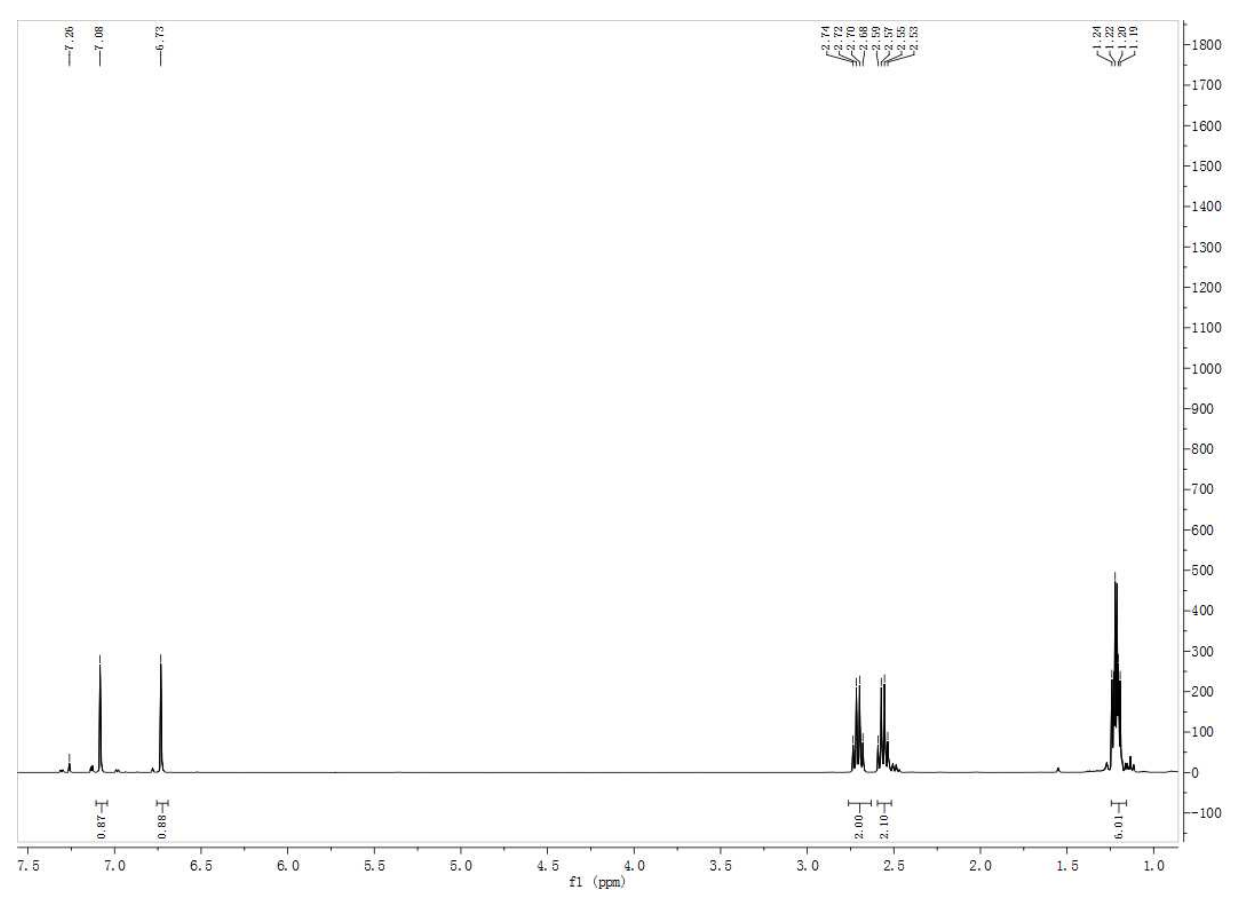

Figure S1c. ${ }^{1}$ H NMR spectrum of compound IT-2. 


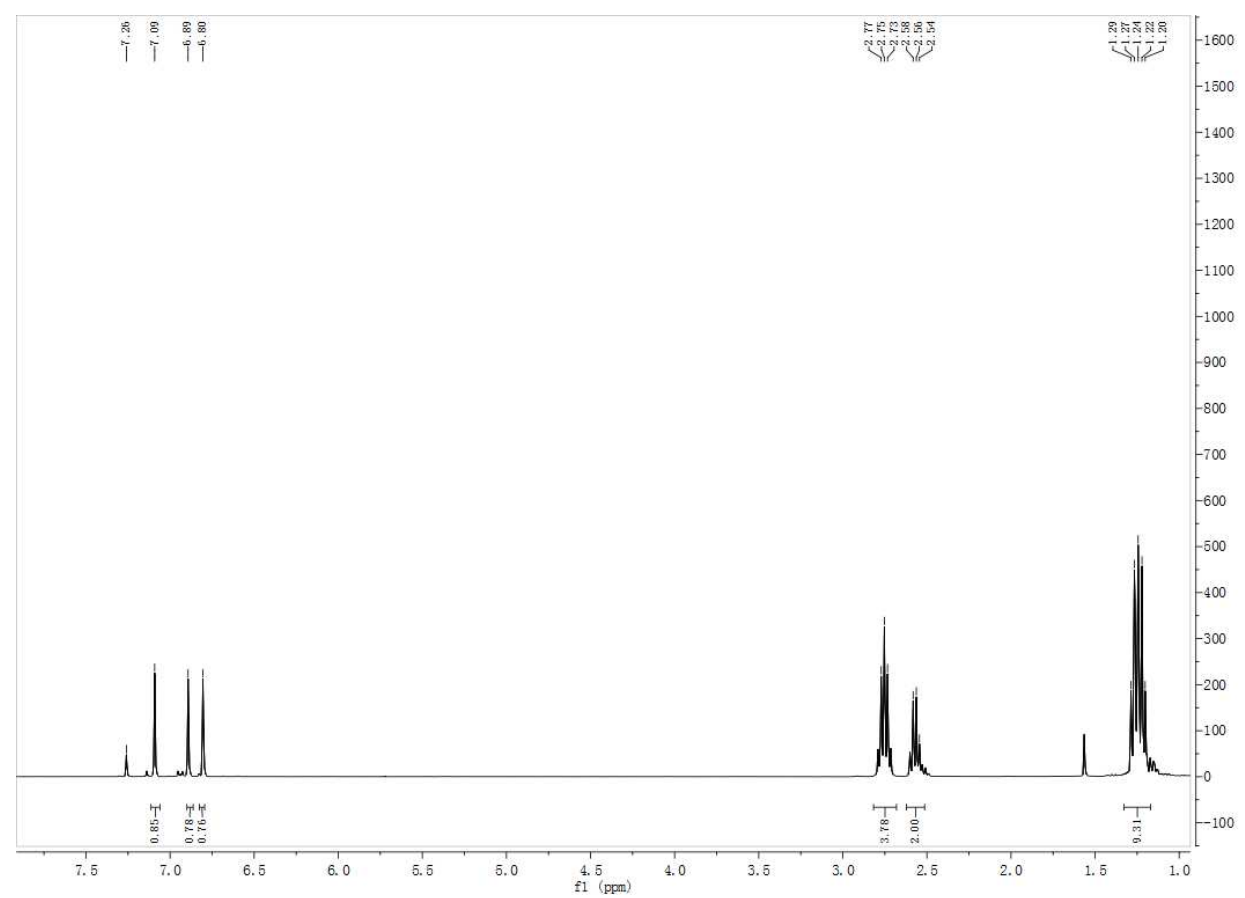

Figure S1d. ${ }^{1} \mathrm{H}$ NMR spectrum of compound IT-3.

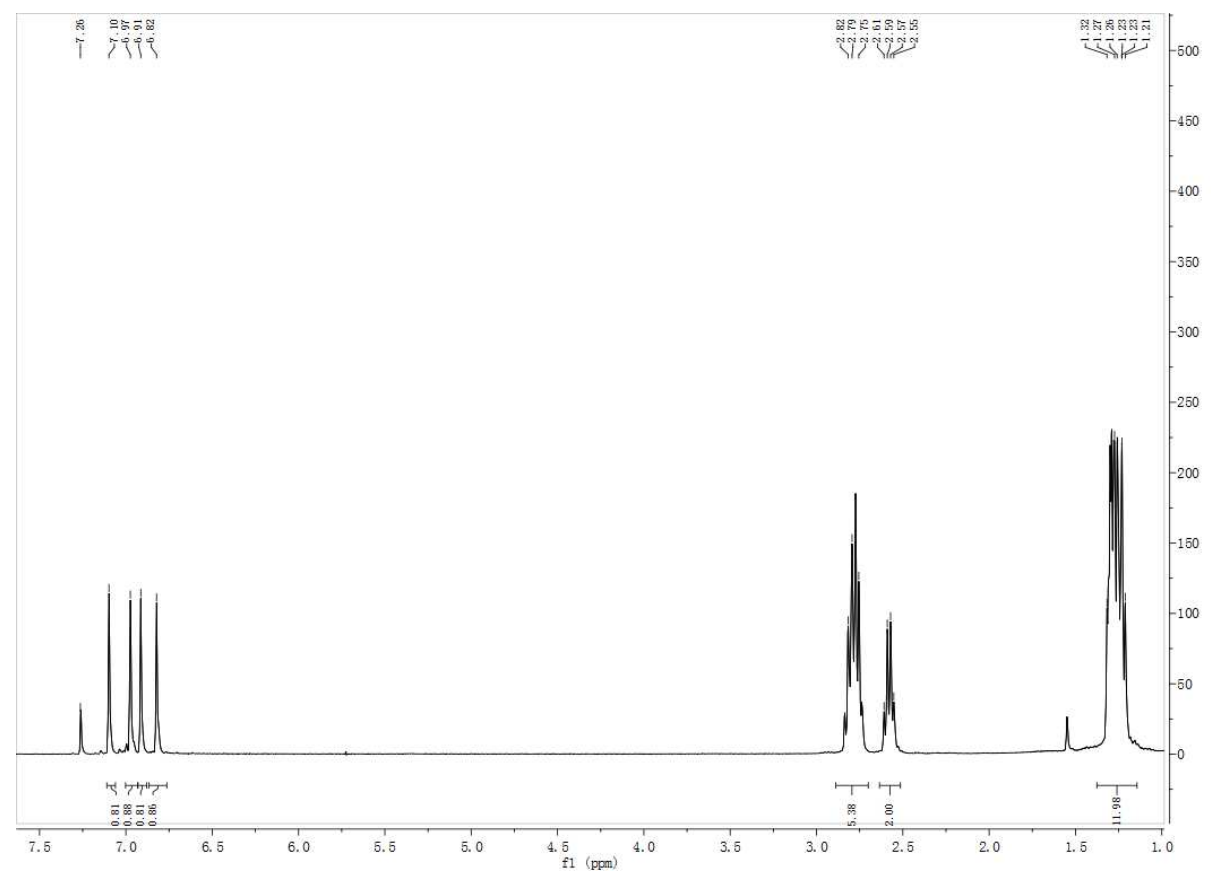

Figure S1e. ${ }^{1} \mathrm{H}$ NMR spectrum of compound IT-4. 


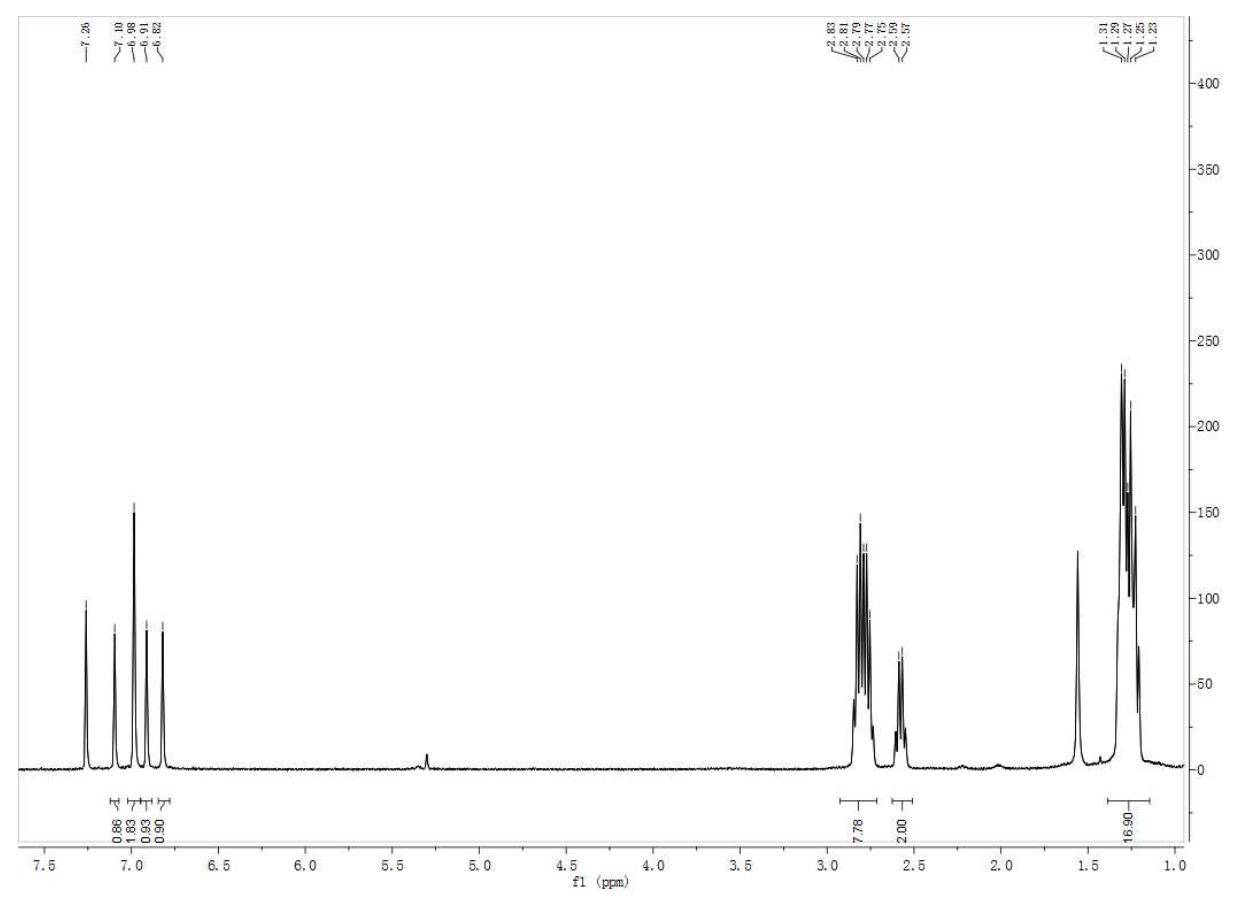

Figure S1f. ${ }^{1} \mathrm{H}$ NMR spectrum of compound IT-5.

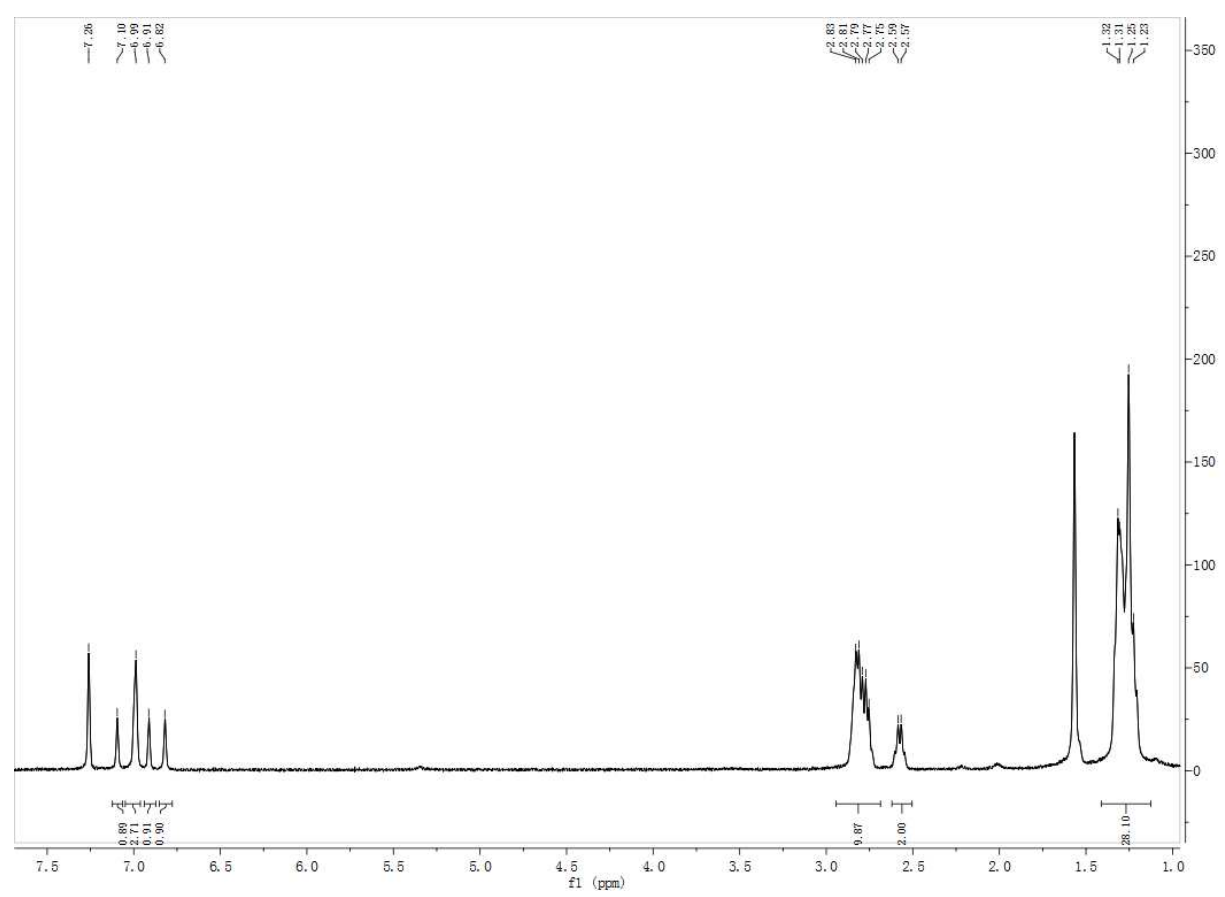

Figure S1g. ${ }^{1} \mathrm{H}$ NMR spectrum of compound IT-6. 
2. Temperature dependent experiments for IT-3 and IT-5.

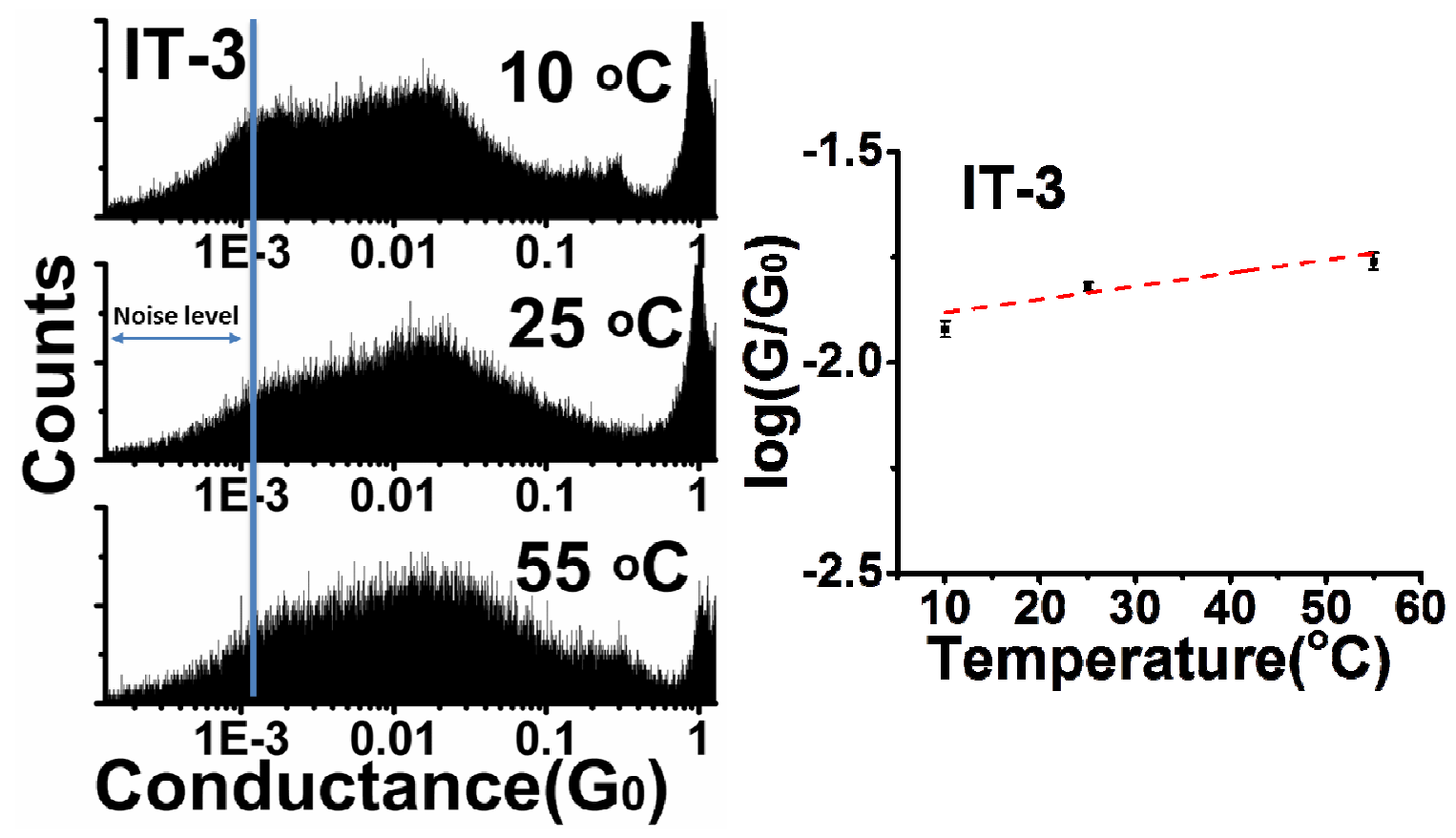

Figure S2a: Conductance histograms and conductance values for IT-3 versus temperature.

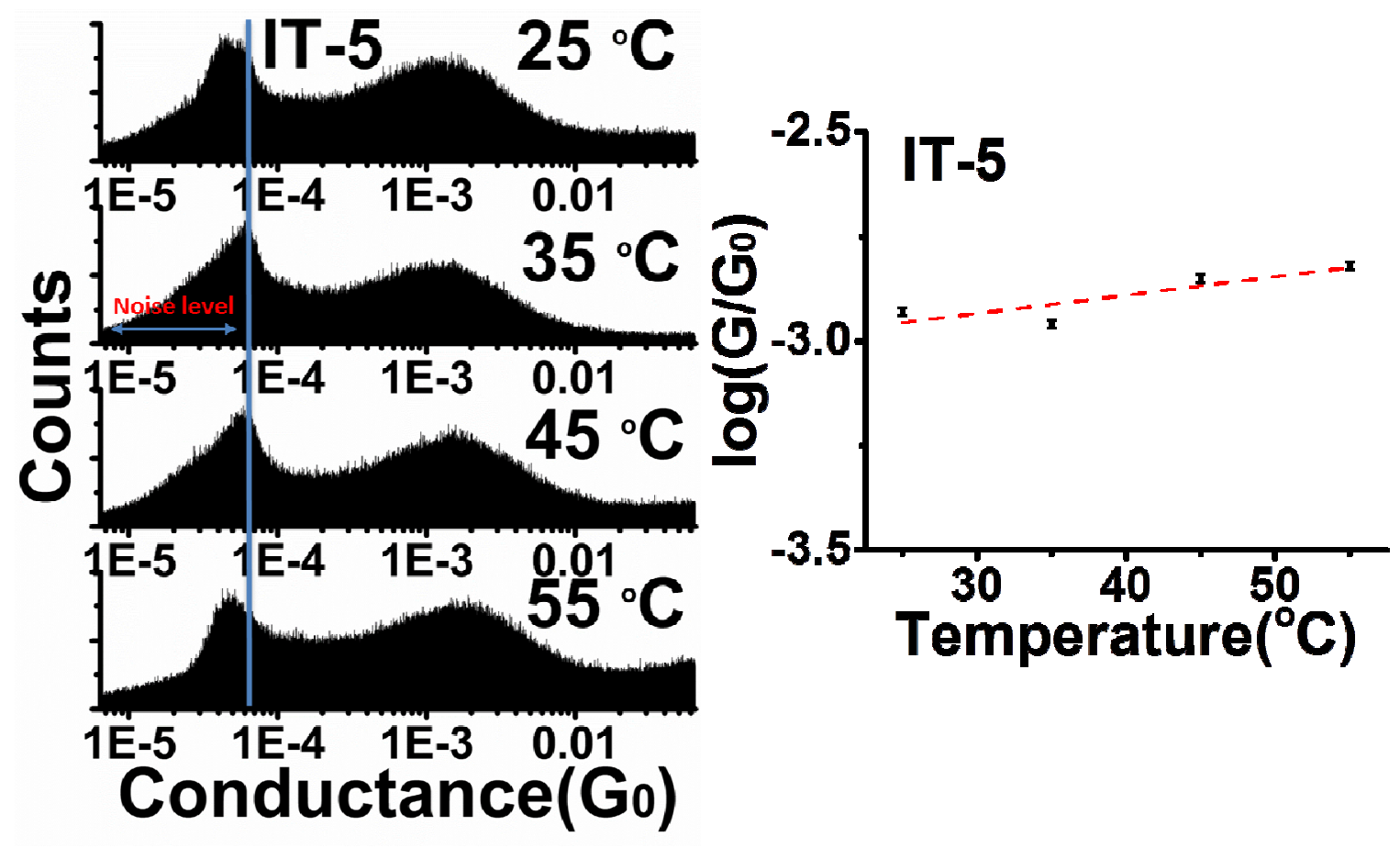


Figure S2b: Conductance histograms and conductance values for IT-5 versus temperature. The peak at around 5E-5 is due to the current at noise level. This was also observed in the blank experiments (see figure S4a).

3. Data collections, 2-Dimensional GV (conductance-voltage, G=I/V) and TVS (Transition voltage spectroscopy) Histograms
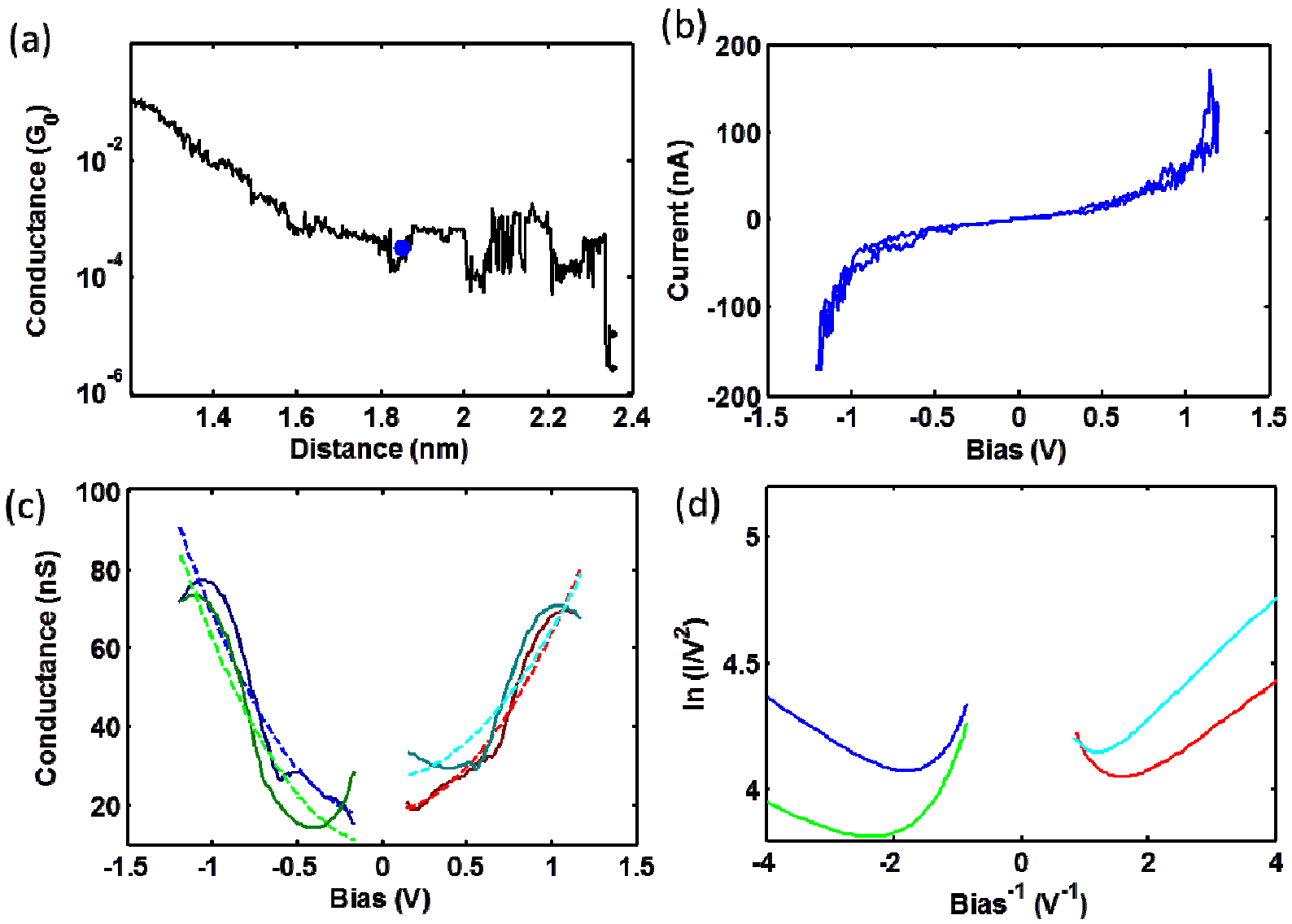

Figure S3a: Illustration of how transition voltage statistics are measured for IT-6. (a) A plateau was detected in the current-distance (I-d) trace during the retraction of the STM tip. STM tip was then stopped at the blue data point. (b) A current-voltage trace is recorded in real time. (c) The IV sweep is then replotted as a GV sweep $(\mathrm{G}=\mathrm{I} / \mathrm{V})$ and fitted to a second order polynomial. (d) 
The polynomial fit is then plotted in a $\ln (\mathrm{G} / \mathrm{V})$ vs. $1 / \mathrm{V}$ plot. After the IV sweep, the STM tip resumes retracting in (a). For more details on the methods, please see our previous work by Guo et al. on J. Am. Chem. Soc. 2011, 133, 19189-19197.

In this method, we modified our STM-Break Junction algorithm to build a histogram of the current transient in real time in order to detect steps before the corresponding molecular junction broke. If a step was detected, the piezo modulation was paused and the bias was swept over a range of $+/-1.2$ Volts. Once the bias sweep was completed, the piezo continued to ramp away from the substrate and the break junction cycle was repeated. In this method, approximately 1500 current-voltage traces were recorded for each molecule. Each measured IV curve was subsequently replotted as a GV curve (where $\mathrm{G}$ is defined as the total conductance, $\mathrm{I} / \mathrm{V}$ ), broken into four regions (corresponding to forward/backward sweep and positive/negative bias) and each region was fitted to a second order polynomial. The fitted polynomials of each GV curve were then mapped onto a $\ln (\mathrm{G} / \mathrm{V})$ vs. $1 / \mathrm{V}$ plot and the minima were taken to be the transition voltages $\left(\mathrm{V}_{\text {trans }}\right)$ of the junction. This process of extracting the transition voltage from the currentvoltage trace is illustrated in Figure S3a. The GV polynomial fitting process was used because it provided an effective means of removing fluctuations from recorded measurements and therefore provided a better estimate of the transition voltage. A more convenient way to extract the transition voltage is to compile the 2-D TVS histogram onto the voltage axis. Then a Gaussian distribution fitting was applied and the peak position indicates the $V_{\text {trans }}$ (See figure S3b-S3f). 1D Conductance histogram at one specific bias voltage can also be extracted from the 2-D G-V histogram (See figure S3b-S3f). Conductance values for IT-n at $0.1 \mathrm{~V}$ bias voltage (see figure $\mathrm{S} 3 \mathrm{~g}$ ) are close to the values in figure $2 \mathrm{~b}$ given by thousands of current-distance traces, ruling out 
the possibility that the STM tip stretching causing the molecule being extended can have a significant impact on the conductance.
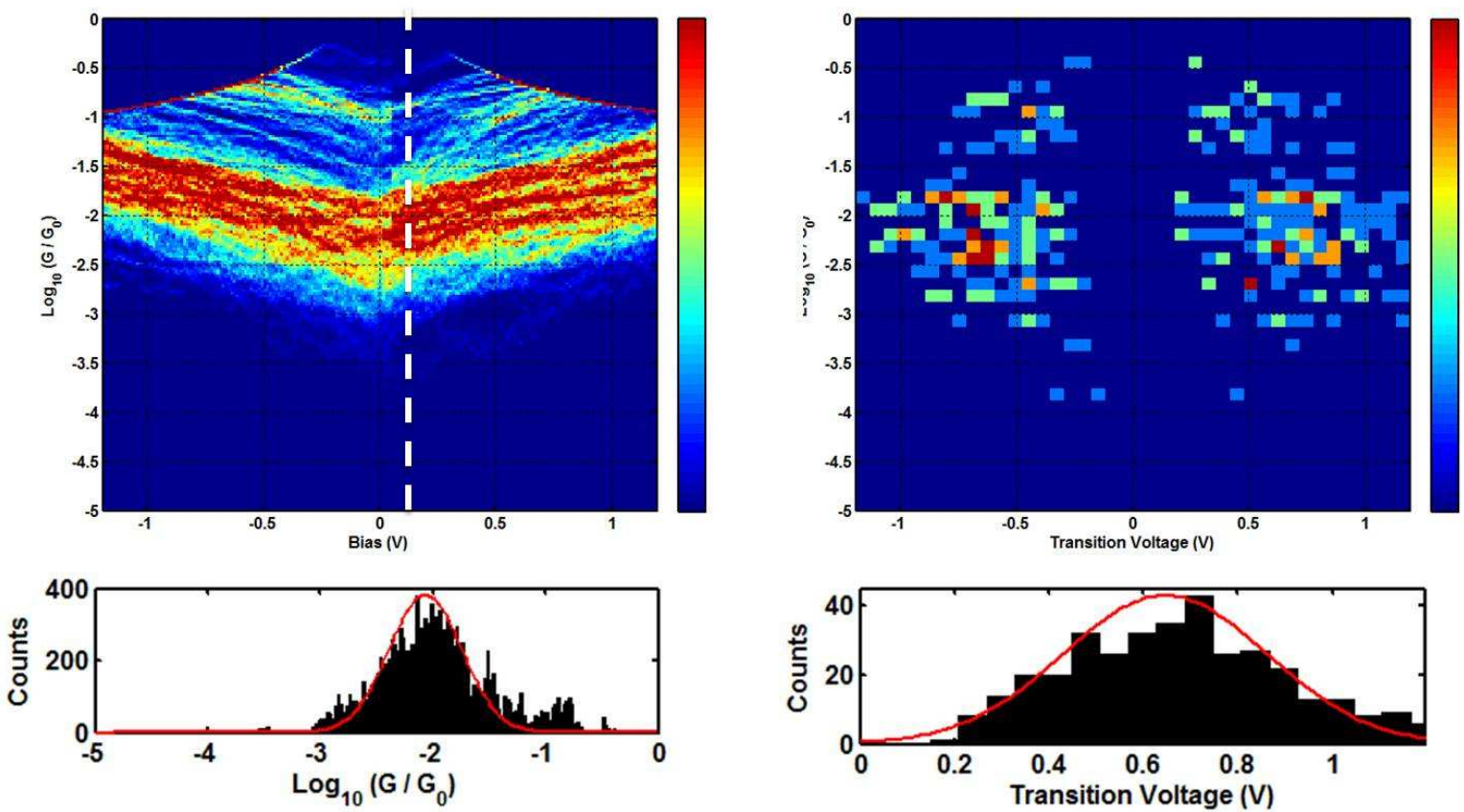

Figure S3b: Two-dimensional GV and TVS histograms for Compound IT-2.
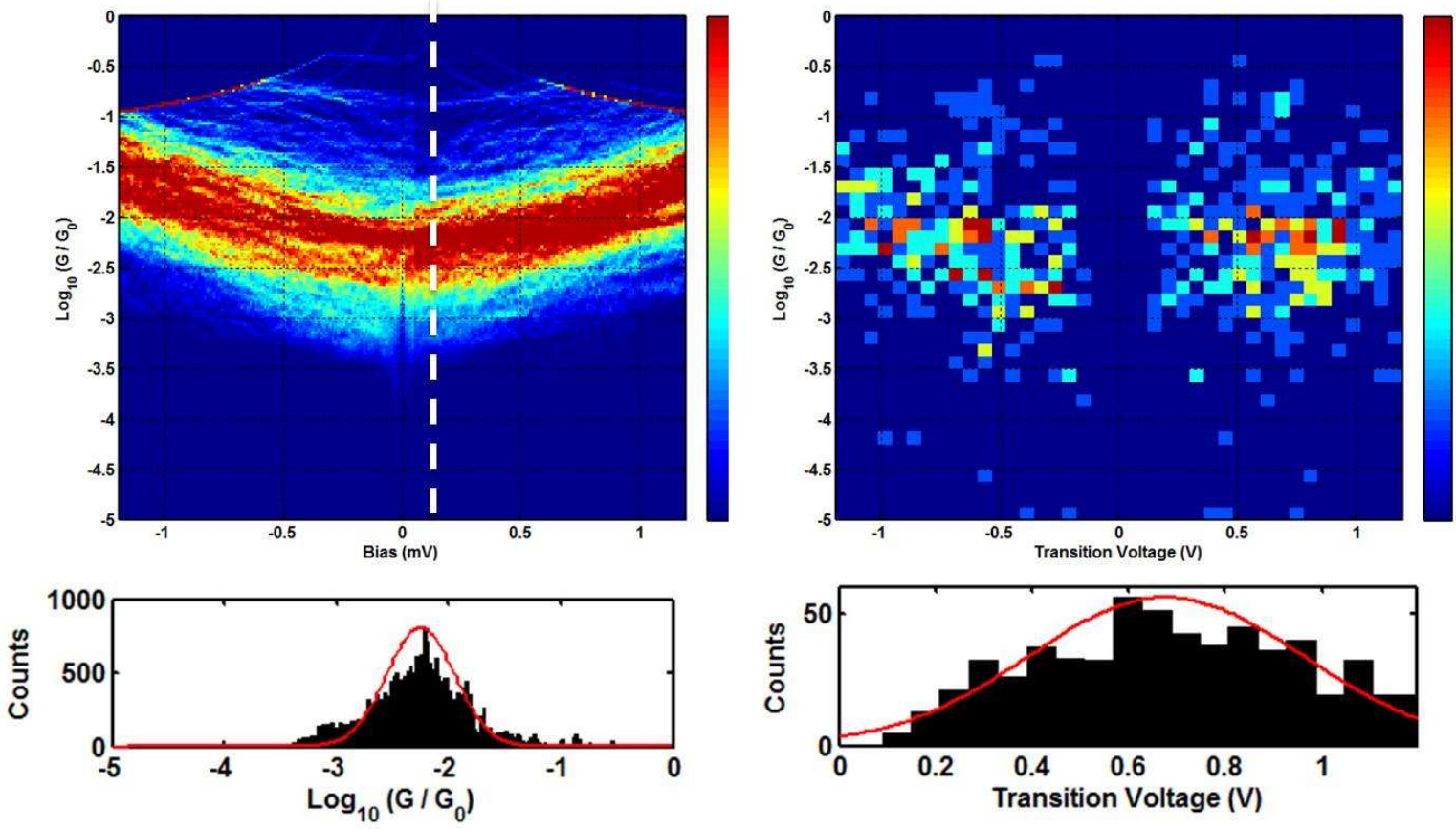
Figure S3c: Two-dimensional GV and TVS histograms for Compound IT-3.
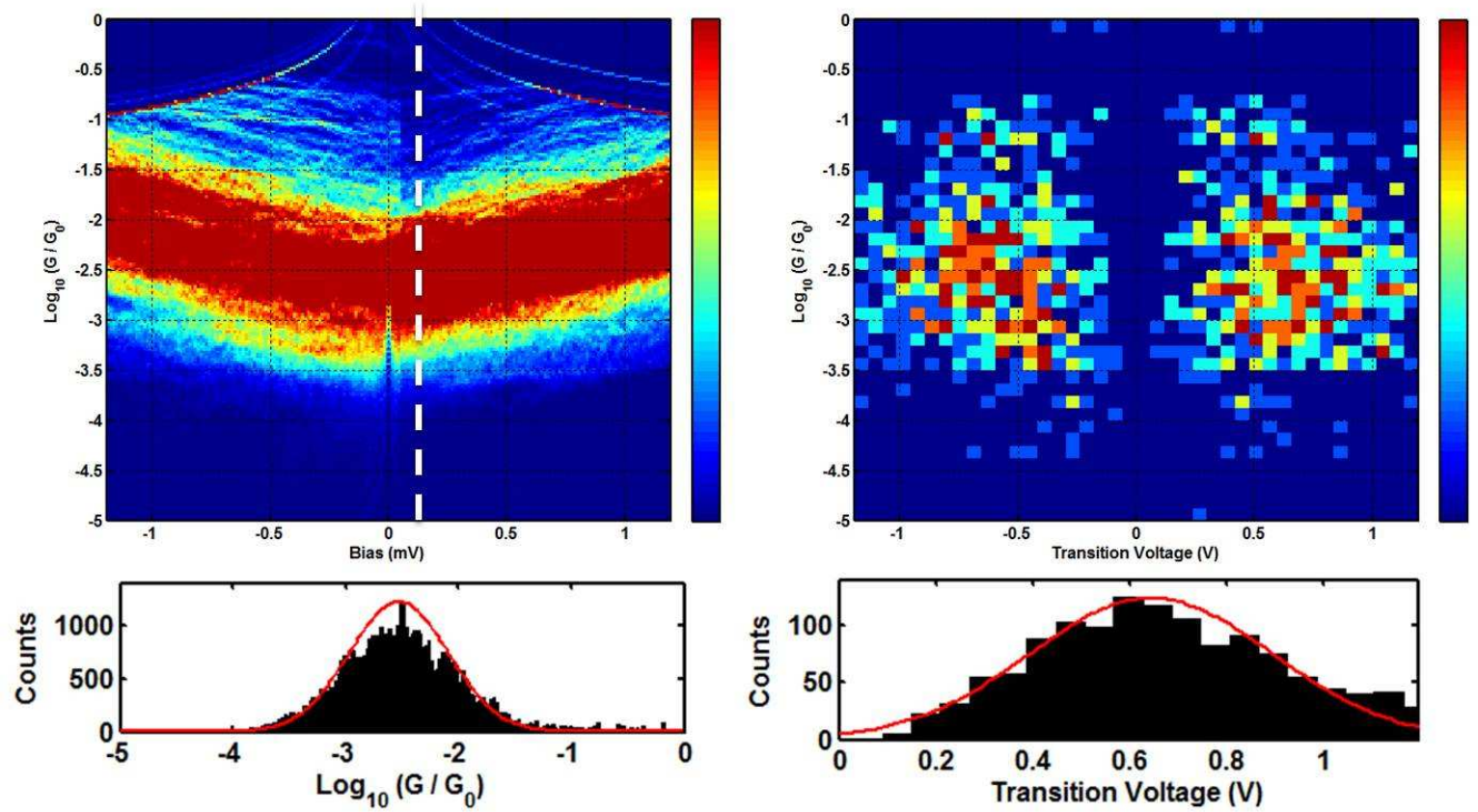

Figure S3d: Two-dimensional GV and TVS histograms for Compound IT-4.
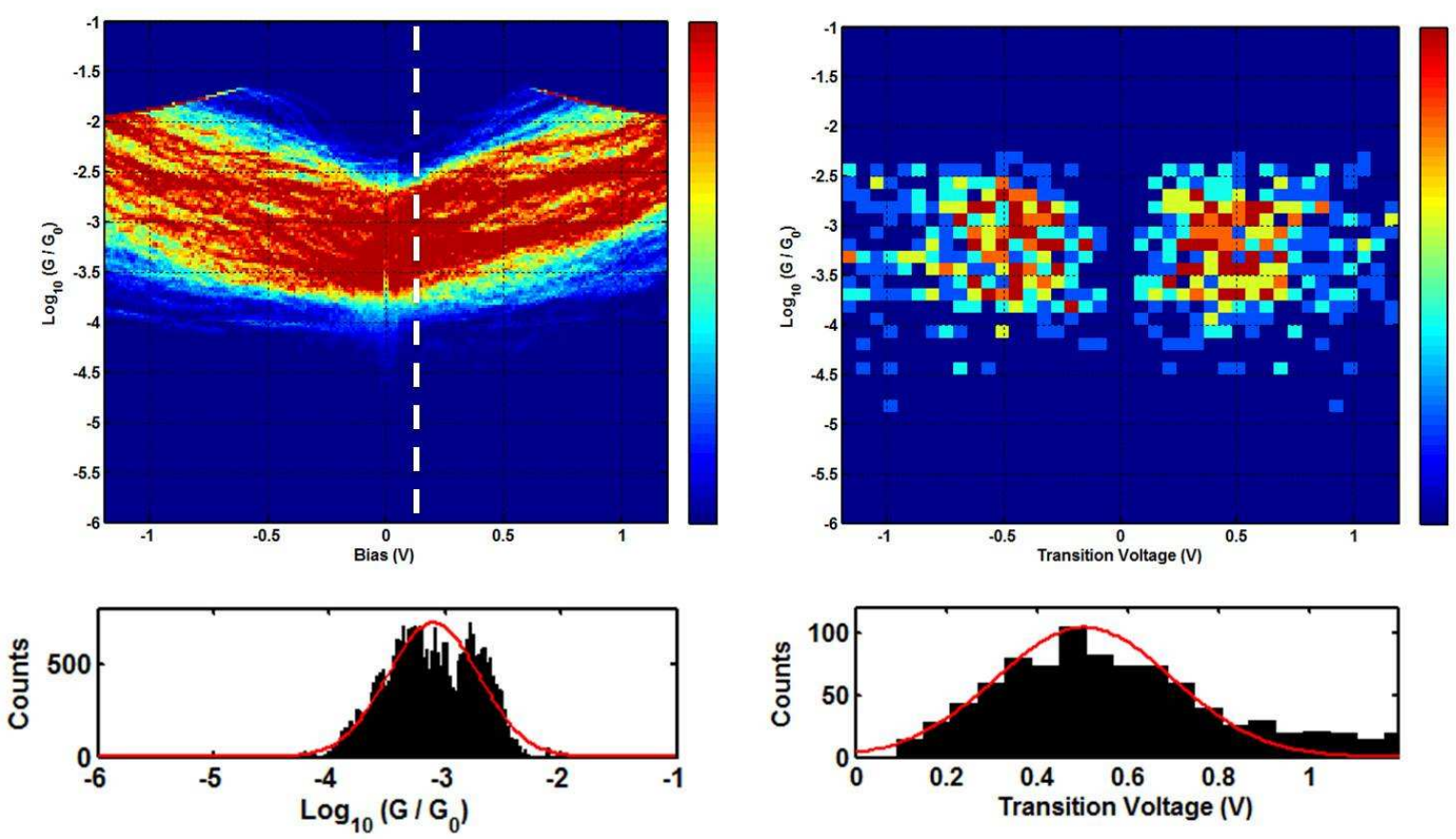

Figure S3e: Two-dimensional GV and TVS histograms for Compound IT-5. 

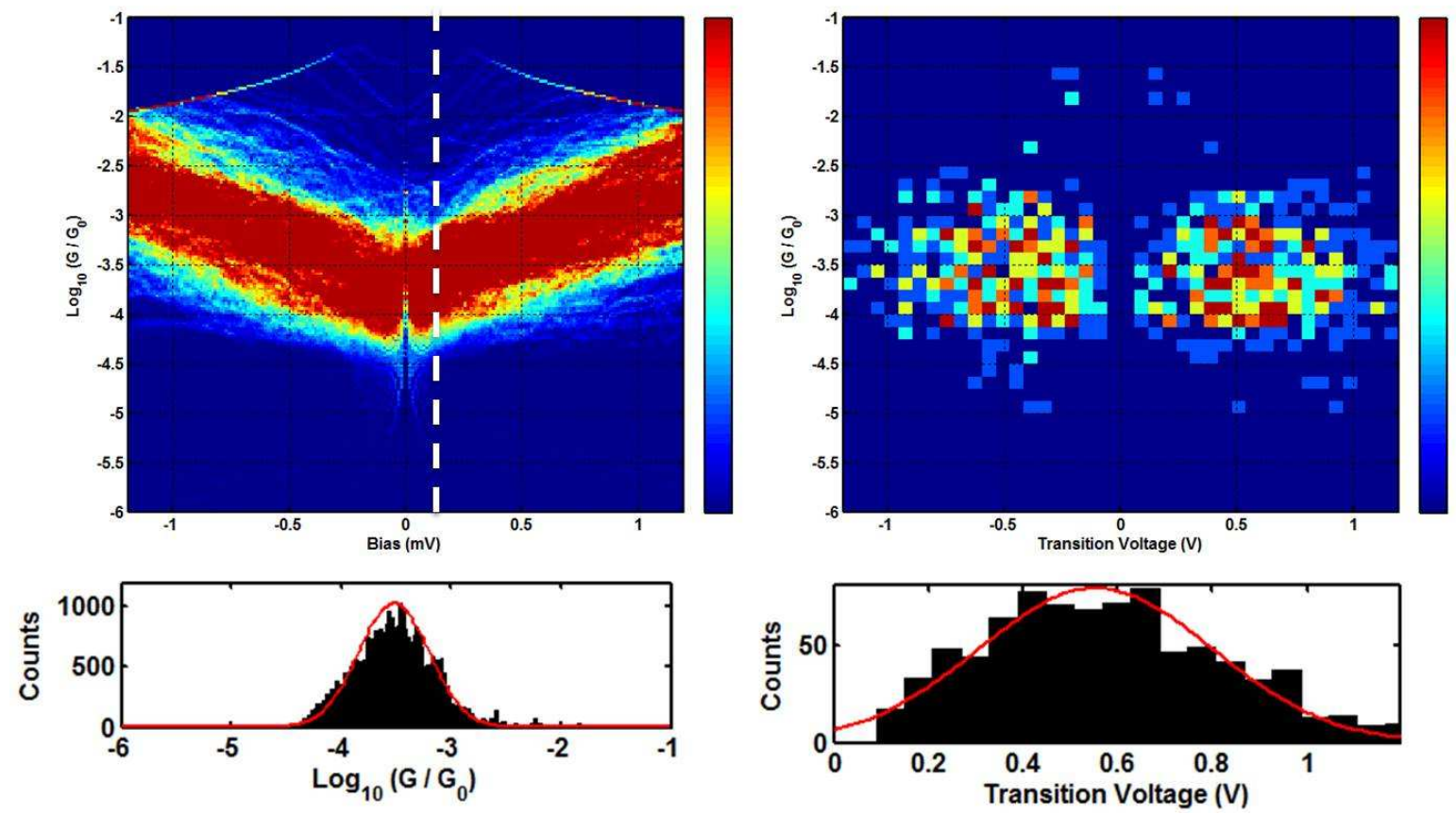

Figure S3f: Two-dimensional GV and TVS histograms for Compound IT-6.

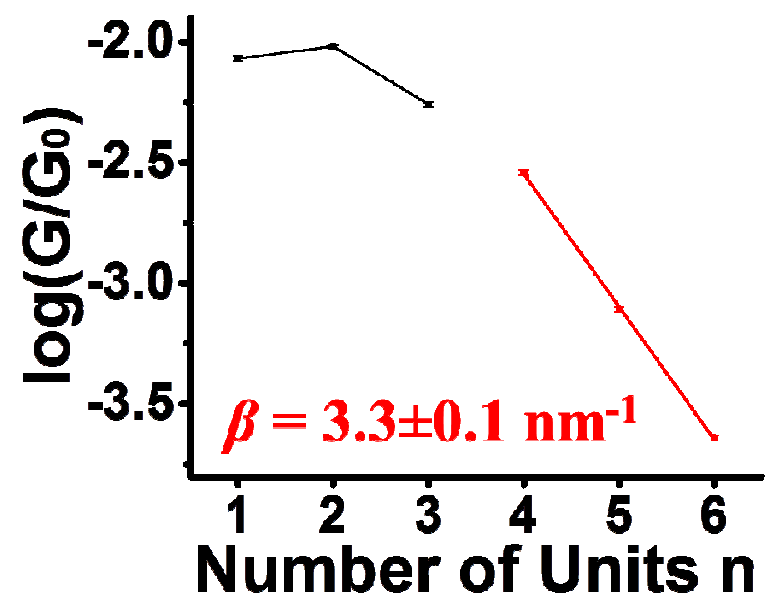

Figure S3g: length dependence plot of conductance at $+0.1 \mathrm{~V}$ bias voltage obtained from 2-D GV histograms (black squares) for IT-n. The conductance trend behaves similarly to the trend in figure $2 \mathrm{~b}$. Error bars are the fitting errors in the Gaussian fitting of the conductance histograms.

\section{Control experiment in mesitylene}



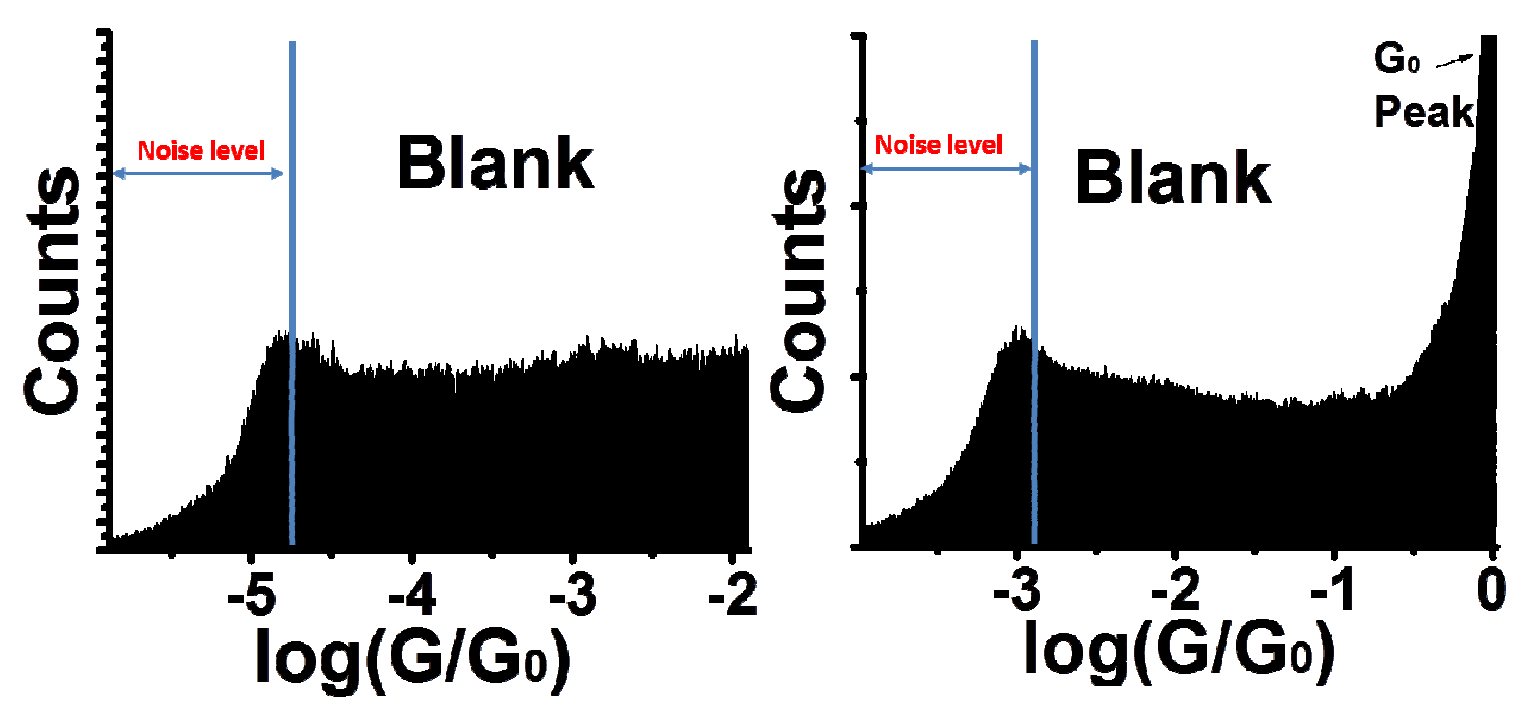

Figure S4a: Conductance histogram for mesitylene with a bias voltage of $100 \mathrm{mV}$ and a gain of $10 \mathrm{nA} / \mathrm{V}$. The shoulder is due to the current at noise level. Notice that different measurable conductance range can be achieved via different gain. The shoulder position also depends on bias voltage and gain, indicating it is due to the noise current from the circuits, not from the solvent.

5. XPS analysis of IT-1 and mesitylene on a Au surface.

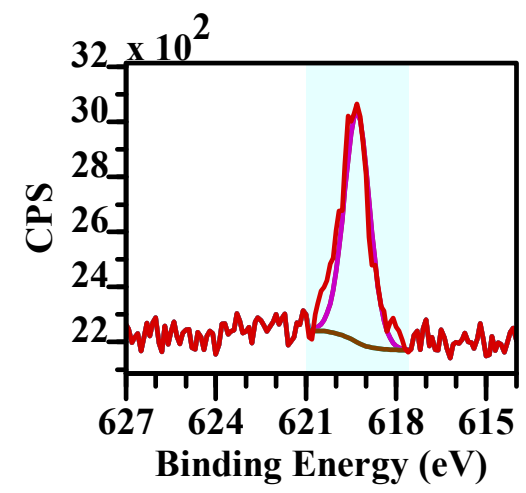

Figure S5a: XPS of IT-1 on Au surface, showing a singlet peak around $619.5 \mathrm{eV}$. 


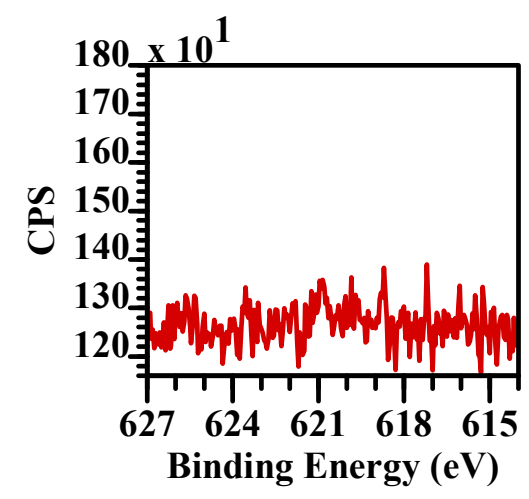

Figure S5b: XPS of mesitylene on Au surface, no peaks in this regime.

\section{Discussions on the possibility of C-I bond cleavage for IT-n.}

We performed ${ }^{1} \mathrm{H}$ NMR and UV-Vis absorption measurements for IT-4 solution before and after its interaction with Au. Gold powder was added into the solution of IT-4 in $\mathrm{CDCl}_{3}$ and the mixture was stirred overnight. Then the solution was firstly characterized by ${ }^{1} \mathrm{H}$ NMR spectroscopy. As shown in Figure S6a, the four proton signals at $\delta=6.82$ to $7.10 \mathrm{ppm}$ with the integrated area ratio of 1:1:1:1 are assigned to the four protons at the $\beta$-position of the four thiophene rings, consistent with the original ${ }^{1} \mathrm{H}$ NMR spectrum of iodo-4mer (Figure S1e) before reacting with gold. No obvious signal assigning to the proton at the $\alpha$-position of thiophene oligomer can be observed, indicating that no iodo groups are cleaved by gold. Moreover, the solution of IT-4 before and after reacting with gold was characterized by UV-Vis absorption spectroscopy. As shown in Figure S6b, the solution of IT-4 displays a maximum absorption at $\lambda$ $=382 \mathrm{~nm}$. Upon stirring with gold powder overnight, no significant difference can be observed for either the absorption maximum or the shape of the absorption spectrum. 


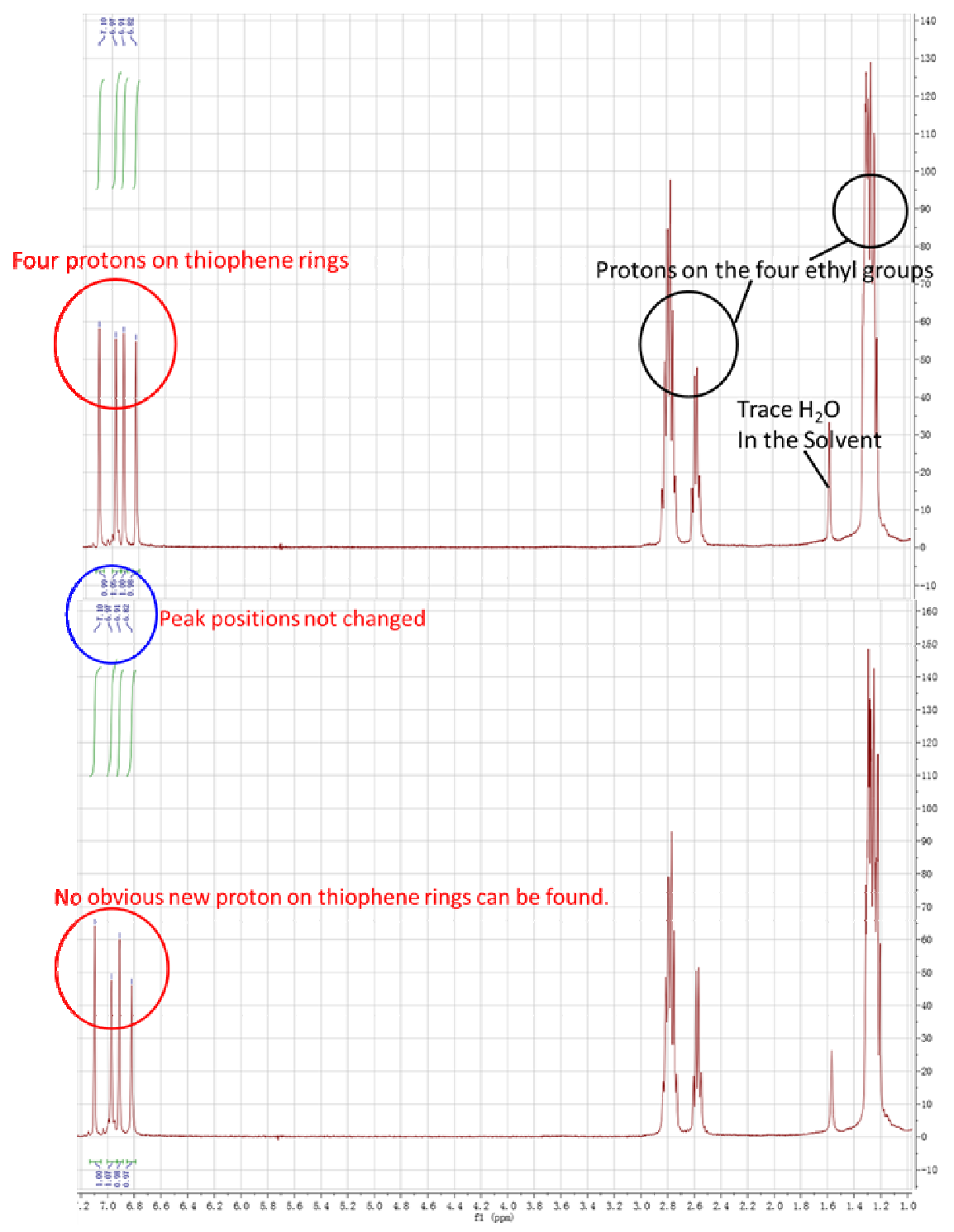

Figure S6a: 1H-NMR of IT-4 before and after reacting with gold powder. 


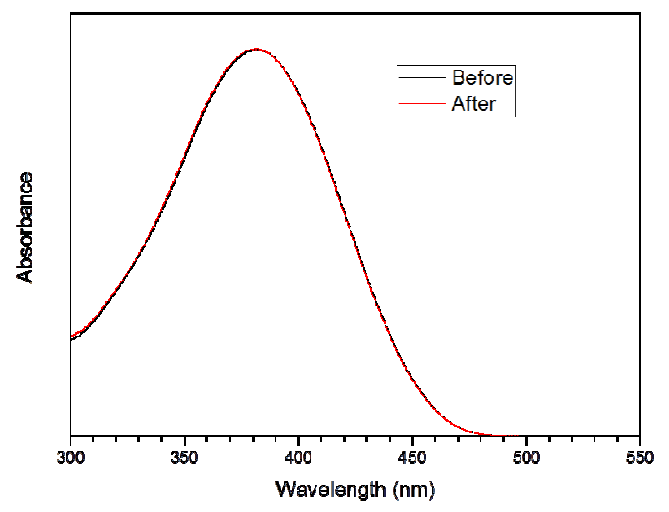

Figure S6b: UV-Vis spectroscopies of IT-4 before and after reacting with gold powder.

Electrochemical cyclic voltammetry was performed on an Autolab system. The sample preparations are the same with the samples for XPS. The gold film served as the working electrode and a Pt wire as the counter electrode. The potential of the gold was controlled with respect to a Ag wire quasi-reference electrode with an Autolab potentiostat. Data collection was performed by GPES software. Ten scan cycles were taken for each of the samples at the scan rate of $50 \mathrm{mV} / \mathrm{s}$. Final results were calculated by averaging the data from the ten scan cycles.

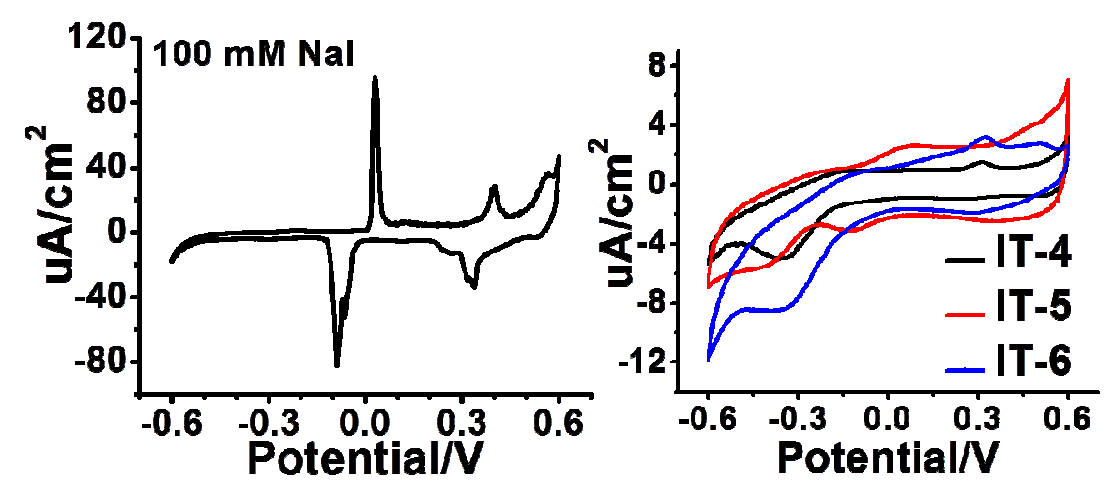

Figure S6c: Cyclic voltammograms of $100 \mathrm{mM} \mathrm{I}^{-}$solution on Au surface versus IT-4, IT-5 and IT-6 modified Au surface in $0.1 \mathrm{KCl}$ solution containing $10 \mathrm{mM}$ phosphate buffer $(\mathrm{pH}=7.0)$. No indication of $\mathrm{I}^{-}$on the Au surface. Cathodic peak around $-0.3 \mathrm{~V}$ is due to oxygen in the air. 


\section{Conductance measurement under nitrogen atmosphere for IT-2}

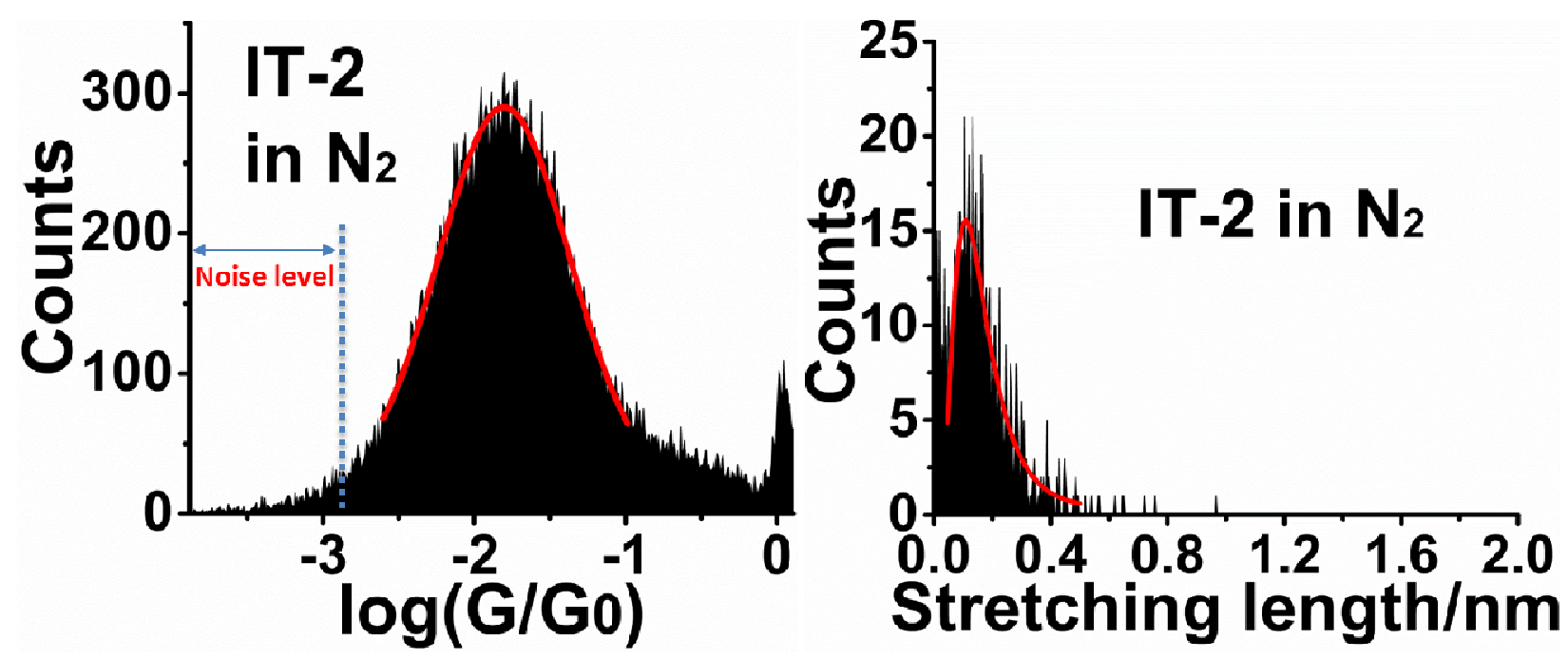

Figure S7: Conductance histogram and stretching length histogram in dry mesitylene under nitrogen atmosphere for IT-2. No differences were seen. Mesitylene was dried by silica gel overnight.

\section{Energy barrier for Rotation of Thiophene Rings.}

To get a better insight of the energy barriers which need to be overcome to reach conformations with higher degree of $\pi$-conjugation, we performed quantum chemical calculations using DFT (B3LYP/ 6-311G**) and IT-3 as a representative system. The energy profile was obtained by performing a molecular relaxation fixing the torsional angle between the terminal and middle thiophene ring. The scan was performed increasing the angle by $5.0^{\circ}$. The most stable conformation has a torsion angle $=-135.5^{\circ}$. The conformation at $=-180.0^{\circ}$ presents an energy barrier of slightly less than $0.05 \mathrm{eV}$. This indicates that such confirmations could be easily achieved at room temperature and even though the transport mechanism is temperature 
independent. The conformational features of the oligothiophenes could be greatly affected by the temperature.

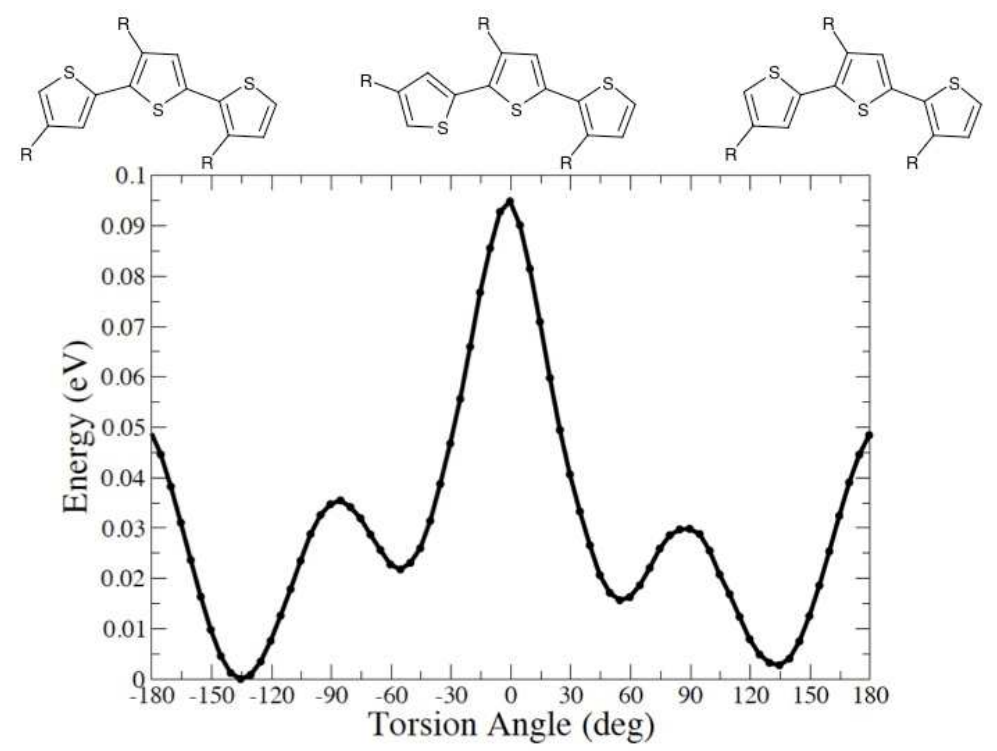

Figure S8: Energy profile of the terminal thiophene unit rotation in the representative system IT-3. The lowest energy conformation has been assigned to $0 \mathrm{eV}$ and $\mathrm{R}=\mathrm{C}_{2} \mathrm{H}_{5}$.

\section{Discussions on the binding geometry of IT-3}

The peak in the conductance histogram of IT-3 can be fitted with two Gaussian distribution, regardless of the bias voltage (figure S9a). Meanwhile, the stretching length of IT-3 is between the shorter stretching length and longer stretching length, and the optimized binding geometry calculation shows that IT-3 molecules start to lift up from the Au surface (figure S9b). This indicates possibly two binding geometries co-exist for IT-3. However, the XPS of IT-3 does not give two distinct peaks in $\mathrm{I}_{5 / 2}$ regime (figure $\mathrm{S} 9 \mathrm{c}$ ). This inconsistent is possibly due to that the release of one iodo group from the Au surface can only occur under the assistance of the STM Au tip. 


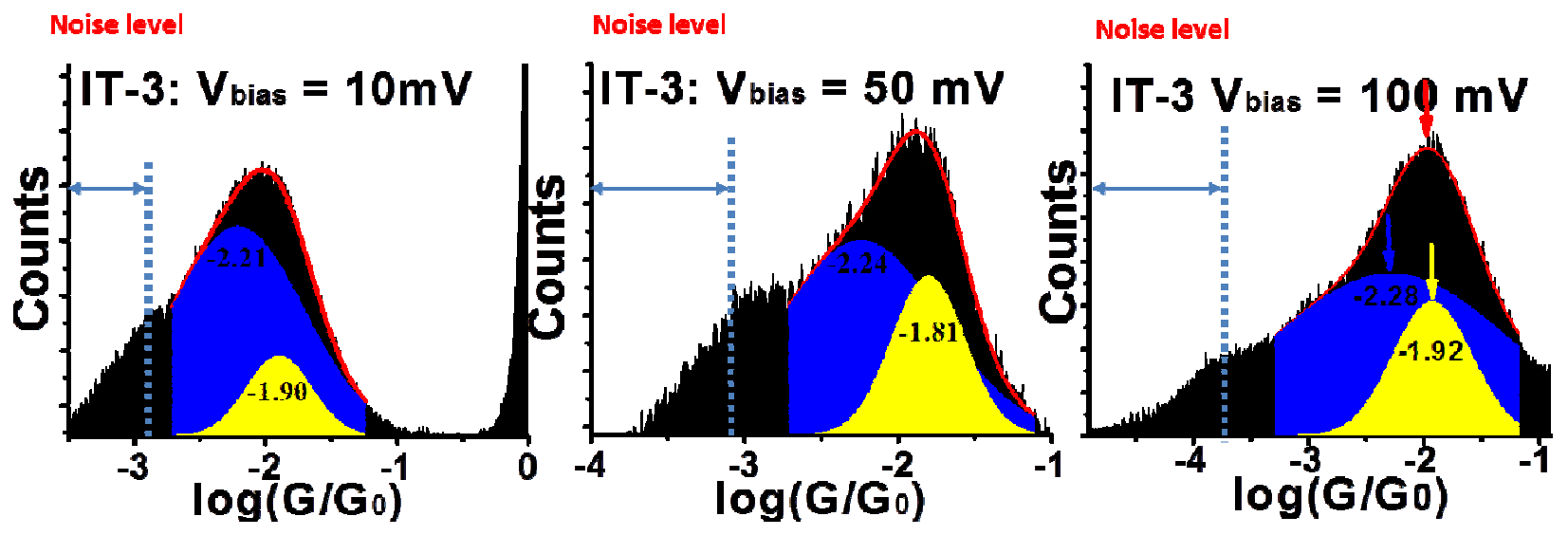

Figure S9a: Conductance histogram for IT-3 under different bias voltage, showing the robustness of the two-peak feature. The conductance histogram of IT-3 was fitted by a two Gaussian distribution, giving a broader peak (blue area) similar to the peak of IT-2 and a sharper peak (yellow area). Arrows indicate the peak positions.

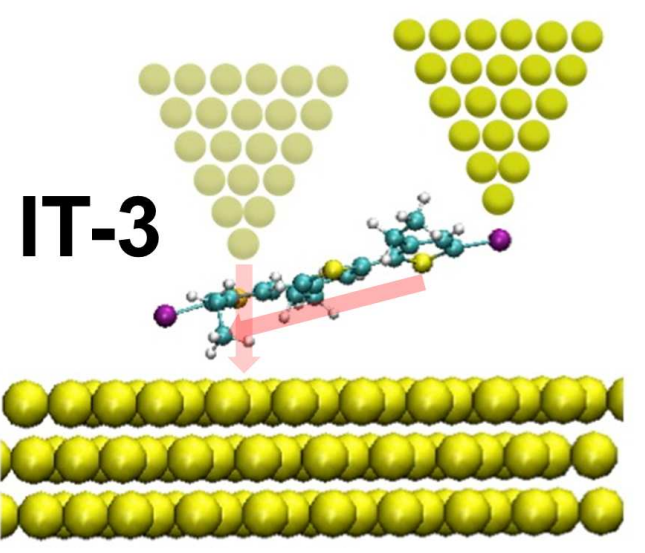

Figure S9b: Calculations of the optimized geometry of IT-3 on a Au $(1,1,1)$ surface 


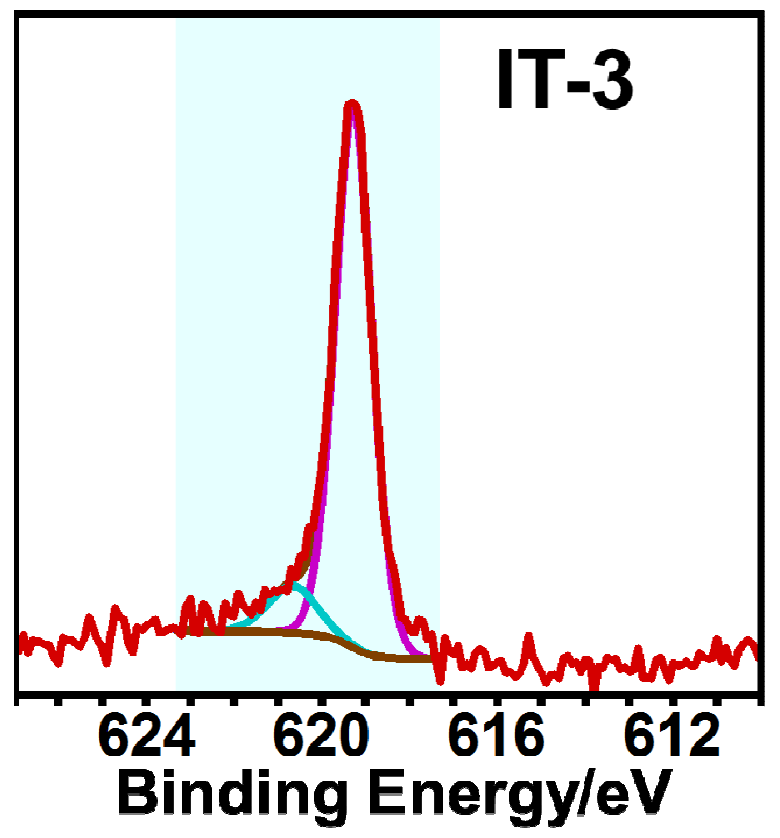

Figure S9c: XPS analysis in $\mathrm{I}_{5 / 2}$ regime of Au surfaces modified by IT-3, showing a peak at $619.3 \mathrm{eV}$ and possibly a shoulder at $620.9 \mathrm{eV}$. 